\title{
Determinate pigeon pea [Cajanus cajan (L) Millsp] genotypes on the north and south coasts of Puerto Rico'
}

\author{
Abad Morales, Brunilda Luciano, Francisco H. Ortiz \\ and Nabor Mendoza ${ }^{2}$
}

\begin{abstract}
Seven experiments were established at the Juana Diaz and Isabela agriculfural experiment substations to evaluate the performance of determinate pigeon pea [Cajanus cajan (L.) Millsp] genotypes. Significant differences were found for yield, height, flowering date, seed weight and number of seeds per pod in almost all the experiments. Many genotypes performed better than the commercial cultivar 2B-Bushy used as check.
\end{abstract}

\section{RESUMEN}

Genotipos determinados de gandules (Cajanus cajan) en las cosias norte y sur de Puerto Rico

En las Subestaciones de Juana Díaz e Isabela se establicieron siete experimentos, en los cuales se evaluaron varios genotipos de gandules de porte determinado. Se encontraron diferencias significativas para rendimiento, altura, fecha de floración, peso de la semilia y número de semiltas por vaina. Se encontraron varios genotipos superiores a la variedad comercial 2B-Bushy. Algunos de estos genotipos se podrían recomendar para siembras comerciales en Puerto Rico.

\section{INTRODUCTION}

Pigeon pea is one of the most important legume crops in the world (5). It is a good source of protein and iron $(3,6,7)$. It is noted for its great adaptability to a wide range of climatic and soil conditions in the tropies. It does well in shallow, poor marginal soils. However, better yields are obtained in deep rich sandy loams. It tolerates drought and high temperatures. In Puerto Rico, pigeon pea is second to beans in importance. Production during 1985-86 was approximately 2,640 tons with a farm value of $\$ 3.1$ million. Demand is higher than local production; therefore, it is also imported mainly from the Dominican Republic. The production of fresh pigeon pea must increase if we want to reduce imports. 
This study was conducted to evaluate the performance of determinate genotypes of pigeon pea at two locations in Puerto Rico.

\section{MATERIALS AND METHODS}

Four field experiments were conducted at the Juana Diaz agricultural experiment substation and three at the Isabela agricultural experiment substation in 1979-80 and 1981-82. The soil at Juana Díaz is a Fraternitad clay (very fine, montmorillonitic, isohyperthermic Udic Chromustert), whereas the soil at Isabela is a Coto clay (clayey, kaolinitic, isohyperthermic Tropeptic Haplorthox). Yield plots consisted of two rows $6.1 \mathrm{~m}$ long with $0.91 \mathrm{~m}$ between rows. Plants were spaced $30 \mathrm{~cm}$ apart in the row. Sencor was applied as a preemergence herbicide at a rate of $1.7 \mathrm{~kg} / \mathrm{ha}$. Plants were sprayed with Lannate $90 \mathrm{wsp}$ at $0.60 \mathrm{~kg} / \mathrm{ha}$ to control podborers. No fungicide nor fertilizer were used. The field was irrigated as needed.

Determinate genotypes tested were obtained from the pigeonpea breeding program of the Puerto Rico Agricultural Experiment Station. Cultivar 2B-Bushy was included in all the experiments as check. Separate analyses of variance were conducted for each year crop because the number of genotypes varied in the different experiments. (Different statistical designs were also used in some experiments.) Fisher L.S.D. at 0.05 was used to compare means (8). Data of the genotypes included in all the experiments by location are presented in the tables.

Thirty-two genotypes were planted at Juana Díaz 10 August and 3 October 1979. Thirty-nine genotypes were planted at Isabela 30 July 1979. A randomized complete block design with four replications was used in all three experiments.

Twenty-seven genotypes were planted at Juana Díaz 4 August 1980, and twenty-two genotypes at Isabela 23 July 1980 . A partially balanced incomplete block design with five replications at Isabela and four at Juana Díaz was used.

Twenty-eight genotypes were planted at Juana Díaz 20 August 1981 and 22 at Isabela 13 August 1981 in randomized complete block designs with four replications. Data on yield, height, days to flower, seed weight and seeds per pod were recorded.

\section{RESULTS AND DISCUSSION}

Tables 1 and 2 present yield of the seven experiments. Mean green pod yield ranged from $7,650 \mathrm{~kg} / \mathrm{ha}$ in $1981-82$ to $9,291 \mathrm{~kg} / \mathrm{ha}$ in the first planting of 1979-80 at Juana Díaz; from $4,995 \mathrm{~kg} / \mathrm{ha}$ in $1981-82$ to 6,177 $\mathrm{kg} / \mathrm{ha}$ in 1980-81 at Isabela. In general, yields were higher at Juana Díaz than at Isabela. This agrees with data obtained by Abrams et al. (1). This difference is expected since soils at Juana Diaz have a higher level of fertility than those at Isabela. Thirteen genotypes yielded better than 
TABLE 1.-Green pod yield (kg/ha) of pigeon pea grown at Juana Diaz, Puerto Rico

\begin{tabular}{|c|c|c|c|c|}
\hline \multirow{2}{*}{ Genotype } & \multicolumn{2}{|c|}{$1979-80$} & \multirow[t]{2}{*}{$1980-81$} & \multirow[t]{2}{*}{$1981-82$} \\
\hline & First planting & Second planting & & \\
\hline 21 & 10645.7 & 10641.2 & 9533.6 & 8822.9 \\
\hline 24 & 5381.2 & 6706.3 & 8107.6 & 4080.7 \\
\hline 30 & 10143.5 & 9275.8 & 8627.8 & 7596.4 \\
\hline 43 & 8080.7 & 8713.0 & 9228.7 & 7802.7 \\
\hline 48 & 6529.1 & 6861.0 & 6582.9 & 6905.8 \\
\hline 49 & 8892.4 & 8704.0 & 7713.0 & 7605.4 \\
\hline 51 & 8959.6 & 8968.6 & 8331.8 & 8439.4 \\
\hline 58 & 9946.2 & 9161.4 & 10107,6 & 7139.0 \\
\hline 69 & 8430.5 & 7204.0 & 9013.4 & 8878.9 \\
\hline 76 & 9838.6 & 7174.9 & 10798.2 & 6591.9 \\
\hline $77-1$ & 13524.6 & 10448.4 & 9443.9 & 7881.2 \\
\hline 86 & 9892.4 & 7787.0 & 9766.8 & 7722.0 \\
\hline 92 & 18816.1 & 8724.2 & 9695.1 & 8278.0 \\
\hline 93 & 11435.0 & 8583.0 & 9443.9 & 8269.0 \\
\hline 97 & 9364.6 & 7269.0 & 8089.7 & 7408.1 \\
\hline 98 & 12627.8 & 7540.4 & 10672.6 & 7201.8 \\
\hline 100 & 8170.4 & 8695.1 & 10885.6 & 6878.9 \\
\hline $100-1$ & 11659.2 & 10946.2 & 9578.5 & 8152.5 \\
\hline 102 & 9363.2 & 8477.6 & 9058.8 & 8376.7 \\
\hline 109 & 11829.6 & 9917.0 & 9766.8 & 8878.9 \\
\hline 110 & 7982.1 & 7542.6 & 8287.0 & 6852.0 \\
\hline 118 & 7479.8 & 8623.3 & 9264.6 & 7148.0 \\
\hline 133 & 8466.4 & 9722,0 & 8852.0 & 7345.3 \\
\hline 135 & 7139.0 & 9009.0 & 9192.8 & 8520.2 \\
\hline 147 & 8591.9 & 8600.9 & 9641.2 & 9845.3 \\
\hline 148 & 8896.9 & 9529.1 & 10152.5 & 7775.8 \\
\hline 2B-Bushy & 7551.6 & 8522.4 & 9506.7 & 7677.1 \\
\hline Mean & 9291.47 & 8485.73 & 9219.72 & 7650.20 \\
\hline L.S.D.(0.05) & 3047.95 & 2423.40 & NS & 1861.23 \\
\hline
\end{tabular}

the mean of the experiment in the first planting of 1979-80 at Juana Diaz. Genotypes 21,77-1,92,93,100-1, and 109 yielded significantly better than 2B-Bushy, the check. In the second planting during the same year 18 genotypes including $2 \mathrm{~B}$-Bushy yielded better than the mean of the experiment, but only 100-1 yielded significantly better than 2B-Bushy. In 198081 no significant difference was found among genotypes. In 1981-82 14 genotypes yielded more than the mean of the experiment, but none yielded significantly higher than 2B-Bushy. Genotype 147 yielded close to the significant level. In general, yields were lower that year at Juana Diaz probably because of environmental conditions. It should be noted that although pigeon pea is tolerant to enviromental stress, higher yields are obtained in favorable conditions. 
TABLE 2,-Green pod yield ( $\mathrm{kg} / \mathrm{ha}$ ) of pigeon pea grown at Isabela, Puerto Rico

\begin{tabular}{cccc}
\hline Genotype & $1979-80$ & $1989-81$ & $1981-82$ \\
\hline 4 & 5830.4 & 6745.4 & 5718.3 \\
29 & 6413.5 & 4861.7 & 4328.0 \\
59 & 5965.0 & 6709.5 & 5404.4 \\
60 & 7086.2 & 6171.3 & 4238.3 \\
65 & 6580.1 & 6278.9 & 5337.1 \\
79 & 7292.5 & 5579.3 & 3946.8 \\
82 & 6171.3 & 7965.3 & 6256.5 \\
83 & 7113.1 & 7229.8 & 5449.2 \\
84 & 7243.2 & 7373.3 & 6413.5 \\
98 & 7220.8 & 5704.9 & 4058.9 \\
$100-1$ & 5471.6 & 5507.5 & 3677.7 \\
109 & 6234.1 & 5256.4 & 3901.9 \\
115 & 5965.0 & 3928.8 & 3341.3 \\
120 & 6395.6 & 6081.6 & 4215.9 \\
134 & 6211.7 & 7660.3 & 6458.3 \\
135 & 5516.5 & 7229.8 & 5815.3 \\
137 & 5543.4 & 6314.8 & 5942.6 \\
147 & 6485.2 & 6243.1 & 5987.4 \\
148 & 6664.6 & 6960.7 & 6278.9 \\
151 & 5830.4 & 6655.7 & 4350.4 \\
153 & 7669.3 & 4987.3 & 4103.7 \\
2B-Bushy & 4798.9 & 4449.1 & 4619.5 \\
Mean & 5904.63 & 6177.04 & 4995.60 \\
L.S.D. & 1758.99 & 1143.54 & 1156.43 \\
\hline
\end{tabular}

At Isabela 7 genotypes yielded significantly better than 2B-Bushy in 1979-80 (table 2). In 1980-81, 14 genotypes yielded significantly better than 2B-Bushy, and in 1981-82 7 genotypes did better than 2B-Bushy. Genotypes 84 and 148 yielded better than 2B-Bushy in the three experiments. Genotypes 98,100-1,109,135,147,148 and 2B-Bushy were included in the 7 experiments; 135,147 and 148 showed more stability, yielding consistently well in all the experiments (table 3). Genotypes 100-1 and 109 did not perform as well at Isabela as at Juana Díaz.

Significant differences were found among genotypes for plant height in the seven experiments (tables 4,5 ). The lowest mean value was obtained in the experiment planted in October at Juana Díaz. Date of planting has a significant effect on plant height in "day-length sensitive pigeon pea" as determined by Abrams and Julia (2). Delayed plantings tend to reduce plant stature. Short genotypes are better suited for mechanical harvesting. Plants one meter high or shorter could be easily harvested mechanically. Genotypes 48 and 21 planted in October at Juana Díaz can be mechanically harvested with a higher efficiency than the other genotypes. 
TABLE 3.-Green pod yield (kg/ha) of pigeon pea grown at Juan Diaz and Isabela, Puerto Rico

\begin{tabular}{|c|c|c|c|c|c|c|c|}
\hline \multirow[b]{2}{*}{ Genotype } & \multicolumn{3}{|c|}{ Juana Diaz } & \multirow{2}{*}{$1981-82$} & \multirow{2}{*}{$\underline{1979-80}$} & \multicolumn{2}{|l|}{ Isabela } \\
\hline & 1st planting & 2nd planting & $1980-81$ & & & & \\
\hline 98 & 12627.8 & 7540.4 & 10672.6 & 7201.8 & 7220.8 & 5704.9 & 4058.9 \\
\hline $100-1$ & 11659.2 & 10946.2 & 9578.5 & 8152.5 & 5471.6 & 5507.5 & 3677.7 \\
\hline 109 & 11829.6 & 9917.0 & 9766.8 & 8878.9 & 6234.1 & 5256.4 & 3901.9 \\
\hline 148 & 8896.9 & 9529.1 & 10152.5 & 7775.8 & 6664.6 & 6960.7 & 6278.9 \\
\hline 2B-Bushy & 7551.6 & 8522.4 & 9506.7 & 7677.1 & 4798.9 & 4449.1 & 4619.5 \\
\hline Mean & 9291.47 & 8485.73 & 9219.72 & 7650.20 & 5904.63 & 6177.04 & 4995.60 \\
\hline L.S.D. $(0.05)$ & 3047.95 & 2423.40 & NS & 1861.23 & 1758.99 & 1143.54 & 1156.43 \\
\hline
\end{tabular}


TABLE 4.-Plant height (m) of pigeon pea grown at Juana Diaz, Puerto Rico

\begin{tabular}{|c|c|c|c|c|}
\hline \multirow[b]{2}{*}{ Genotype } & \multicolumn{2}{|c|}{$1979-80$} & \multirow[t]{2}{*}{$1980-81$} & \multirow[t]{2}{*}{$1981-82$} \\
\hline & First planting & Second planting & & \\
\hline 21 & 1.150 & 1.003 & 1.253 & 1.302 \\
\hline 24 & 1.173 & 1.080 & 1.308 & 1.291 \\
\hline 30 & 1.208 & 0.998 & 1.385 & 1.841 \\
\hline 43 & 1.160 & 1.008 & 1.265 & 1.859 \\
\hline 48 & 1.005 & 1.000 & 1.095 & 1.122 \\
\hline 49 & 1.090 & 1.055 & 1.163 & 1.284 \\
\hline 51 & 1.198 & 1.198 & 1.230 & 1.277 \\
\hline 53 & 1.185 & 1.150 & 1.305 & 1.305 \\
\hline 69 & 1.190 & 0.938 & 1.373 & 1.396 \\
\hline 76 & 1.128 & 0.920 & 1.323 & 1.165 \\
\hline $77-1$ & 1.240 & 0.993 & 1.428 & 1.583 \\
\hline 86 & 1.275 & 1.210 & 1.348 & 1.274 \\
\hline 92 & 1.230 & 1.048 & 1.348 & 1.386 \\
\hline 93 & 1.288 & 1.050 & 1.378 & 1.365 \\
\hline 97 & 1.160 & 1.100 & 1.360 & 1.309 \\
\hline 98 & 1.250 & 1.033 & 1.460 & 1.373 \\
\hline 100 & 1.200 & 1.098 & 1.458 & 1.388 \\
\hline $100-1$ & 1.263 & 0.978 & 1.388 & 1.450 \\
\hline 102 & 1.098 & 1.043 & 1.288 & 1.317 \\
\hline 109 & 1.153 & 1.093 & 1.343 & 1.397 \\
\hline 110 & 1.065 & 1.025 & 1.250 & 1.394 \\
\hline 118 & 1.250 & 1.068 & 1.428 & 1.423 \\
\hline 133 & 1.257 & 1.133 & 1.320 & 1.354 \\
\hline 135 & 1.190 & 1.055 & 1.390 & 1.410 \\
\hline 147 & 1.258 & 1.060 & 1.445 & 1.516 \\
\hline 148 & 1.235 & 1.090 & 1.458 & 1.414 \\
\hline 2B-Bushy & 1.108 & 0.978 & 1.195 & 1.185 \\
\hline Mean & 1.1817 & 1.0535 & 1.3306 & 1.3477 \\
\hline L.S.D. $(0.05)$ & 0.0854 & 0.0973 & 0.1126 & 0.1275 \\
\hline
\end{tabular}

Plants grew taller at Isabela than at Juana Diaz. This may be attributed to the higher amount of rain at Isabela, which is located in the humid region of the island whereas Juana Diaz is in the dry region.

Significant differences between flowering dwarfs were also found among genotypes in the four experiments in which data for this character was recorded (tables 6,7). As in plant height, the lowest mean was found in the October planting at Juana Díaz. This trait is also affected by day length. Flowering date and plant height are highly positively correlated, whereas flowering date is inversely correlated with yield (4). Genotypes flowering significantly earlier than $2 B$-Bushy were found only in the October planting at Juana Diaz $(48,49,51,53,86)$. Apparently these genotypes are affected more by day length. 
J. Agric. Univ. P.R. voL. 72, No. 4, OCTOBER, 1988

TABLE 5.-Plant height ( $m$ ) of pigeon pea grown at Isabela, Puerto Rico

\begin{tabular}{|c|c|c|c|}
\hline \multirow[b]{2}{*}{ Genotype } & \multicolumn{3}{|c|}{ Plant height (cm) } \\
\hline & $1979-80$ & $1980-81$ & $1981-82$ \\
\hline 4 & 1.469 & 1.738 & 1.503 \\
\hline 29 & 1.441 & 1.779 & 1.588 \\
\hline 59 & 1.149 & 1.655 & 1.346 \\
\hline 60 & 1.383 & 1.731 & 1.552 \\
\hline 65 & 1.325 & 1.644 & 1.358 \\
\hline 79 & 1.211 & 1.598 & 1.368 \\
\hline 82 & 1.421 & 1.806 & 1.485 \\
\hline 83 & 1.455 & 1.776 & 1.488 \\
\hline 84 & 1.496 & 1.817 & 1.643 \\
\hline 98 & 1.398 & 1.696 & 1.518 \\
\hline $100-1$ & 1.338 & 1.703 & 1.530 \\
\hline 109 & 1.293 & 1.665 & 1.454 \\
\hline 115 & 1.360 & 1.592 & 1.402 \\
\hline 120 & 1.473 & 1.812 & 1.622 \\
\hline 134 & 1.430 & 1.789 & 1.588 \\
\hline 135 & 1.483 & 1.815 & 1.686 \\
\hline 137 & 1.400 & 1.763 & 1.550 \\
\hline 147 & 1.532 & 1.810 & 1.562 \\
\hline 148 & 1.589 & 1.795 & 1.577 \\
\hline 151 & 1.408 & 1.729 & 1.527 \\
\hline 153 & 1.387 & 1.713 & 1.428 \\
\hline 2B-Bushy & 1.249 & 1.631 & 1,312 \\
\hline Mean & 1.3775 & 1.7299 & 1,4969 \\
\hline L.S.D. $(0.05)$ & .1286 & .0749 & .0889 \\
\hline
\end{tabular}

Significant differences were found for seed weight in 6 experiments in which this trait was evaluated (tables 7,8 ). Seeds at genotype 48 were significantly heavier than those of 2B-Bushy in the four experiments at Juana Diaz; 33 had the lowest seed weight. None of these genotypes were included in the experiments planted at Isabela.

Tables 9 and 10 present data on number of seeds per pod. Significant differences were found for this trait in 6 out of the 7 experiments. However, differences among all genotypes are small. No significant difference was found in 1981-82 at Isabela.

The data show that genotypes better than 2B-Bushy can be selected and recommended for commercial plantings.

\section{LITERATURE CITED}

1. Abrams, R., J. Vélez Fortuño and J. López Garcia, 1969. The interaetion of variety and environment in pigeon pea (Cajanus cajan) trials. J. Agric. Univ. P. R. 53 (1):61-6.

2. ___ and F. J. Julia, 1973. Effect of planting time, plant population and row spacing on yield and other characteristics of pigeon pea (Cajanus cajan (L.) Millsp.). J. Agric. Univ. P. R. 57 (4):275-85. 
TABLE 6.-Days to 50\% flowering of pigeon pea grown at Fortuna, Puerto Rico

\begin{tabular}{|c|c|c|c|}
\hline \multirow[b]{2}{*}{ Genotype } & \multicolumn{2}{|c|}{$\underline{1979-80}$} & \multirow[b]{2}{*}{$1980-81$} \\
\hline & First planting & Second planting & \\
\hline 21 & 97.5 & 74.5 & 95.5 \\
\hline 24 & 97.0 & 72.0 & 92.0 \\
\hline 30 & 97.5 & 77.0 & 98.3 \\
\hline 43 & 96.5 & 77.0 & 90.8 \\
\hline 48 & 96.5 & 67.0 & 89.5 \\
\hline 49 & 97.5 & 68.0 & 96.3 \\
\hline 51 & 96.0 & 64.0 & 91.0 \\
\hline 58 & 96.5 & 67.0 & 92.0 \\
\hline 69 & 98.0 & 78.5 & 100.3 \\
\hline 76 & 110.5 & 72.3 & 112.3 \\
\hline $77-1$ & 118.8 & 78.5 & 120.5 \\
\hline 86 & 96.0 & 66.0 & 95.0 \\
\hline 92 & 111.3 & 73.0 & 116.5 \\
\hline 93 & 112.0 & 77.8 & 116.5 \\
\hline 97 & 119.8 & 73.0 & 115.3 \\
\hline 98 & 116.5 & 73.0 & 115.8 \\
\hline 100 & 119.0 & 73.0 & 117.8 \\
\hline $100-1$ & 118.8 & 75.8 & 116.5 \\
\hline 102 & 116.0 & 73.0 & 114.0 \\
\hline 109 & 114.5 & 73.0 & 115.3 \\
\hline 110 & 123.3 & 78.0 & 118.3 \\
\hline 118 & 99.5 & 79.3 & 95,0 \\
\hline 133 & 96.0 & 87.5 & 97.0 \\
\hline 135 & 98.0 & 74.5 & 103.00 \\
\hline 147 & 100.5 & 78.5 & 102.8 \\
\hline 148 & 104.3 & 77.8 & 99.8 \\
\hline 2B-Bushy & 98.5 & 77.8 & 95.5 \\
\hline Mean & 104.75 & 74.84 & 104.13 \\
\hline L.S.D. $(0.05)$ & 4.47 & 6.06 & 7.27 \\
\hline
\end{tabular}

3. Jambunathan, R. and V. Singh, 1981. Grain quality of pigeon pea. Proceedings of the International Workshop on pigeon peas. ICRISAT, 15-19 Dec. 1980. Patancheru, India. Vol. 1:351-56.

4. Morales-Muñz, A. and R. Abrams, 1971. Inheritance of some quantitative characters in pigeon peas (Cajanus cajan). J. Agric. Univ. P. R. 55 (1):23-43.

5. Morton, J. F., R. E. Smith, M. A. Lugo-López and R. Abrams, 1982, Pigeon peas (Cajanus cajan Milisp): A Valuable crop of the tropics. Special publication of the College of Agricultural Sciences, Department of Agronomy and Solls, Mayagüez, P. R.

6. Parsi-Ros, O., E. J. Rodriguez, J. R. Cruz-Cay and M. F. Cintrón, 1987. Processing and the nutritional contents of canned and not canned pigeon peas (Cojanus cajan (L.) Millsp). J. Agric. Univ. P. R. 71 (1):33-41.

7. ____ _, and, 1987 . Processing and storage and the nutritional contents of pigeon peas (Cajanus cajan (L.) Millsp). J. Agric. Univ. P. R. 71 (1):43-61.

8. Snedecor, G. W. and W. G. Cochran, 1980. Statistical Methods 7th ed., The Iowa State University Press, Ames, Iowa. 
J. Agric. Univ. P.R. voL. 72, No. 4, OCTOBER, 1988

TABLE 7.-Days to $50 \%$ flowering and weight $(g$.) of 100 seeds of pigeon pea groum at Isabela, Puerto Rico

\begin{tabular}{|c|c|c|c|}
\hline \multirow[b]{2}{*}{ Genotype } & \multirow{2}{*}{$\begin{array}{c}\begin{array}{c}\text { Days to } 50 \% \\
\text { flowering }\end{array} \\
1981-82\end{array}$} & \multicolumn{2}{|c|}{ 100-seed weight } \\
\hline & & $1980-81$ & $1981-82$ \\
\hline 4 & 88.0 & 36.1 & 39.8 \\
\hline 29 & 88.0 & 31.4 & 32.0 \\
\hline 59 & 110.0 & 22.5 & 31.6 \\
\hline 60 & 116.8 & 24.0 & 35.0 \\
\hline 65 & 110.0 & 23.2 & 31.2 \\
\hline 79 & 110.0 & 22.8 & 29.6 \\
\hline 82 & 88.0 & 30.5 & 30.9 \\
\hline 83 & 88.0 & 31.5 & 30.6 \\
\hline 84 & 89.8 & 31.7 & 31.1 \\
\hline 98 & 112.3 & 24.9 & 35.5 \\
\hline $100-1$ & 119.0 & 26.8 & 34.3 \\
\hline 109 & 116.8 & 25.0 & 34.5 \\
\hline 115 & 88.0 & 36.3 & 34.8 \\
\hline 120 & 98.3 & 27.6 & 28.4 \\
\hline 134 & 88.0 & 32.5 & 33.0 \\
\hline 135 & 88.0 & 33.9 & 33.2 \\
\hline 137 & 88.0 & 29.8 & 38.7 \\
\hline 147 & 88.0 & 32.1 & 34.7 \\
\hline 148 & 89.8 & 32.2 & 32.6 \\
\hline 151 & 88.0 & 31.3 & 31.8 \\
\hline 153 & 110.0 & 26.6 & 32.2 \\
\hline 2B-Bushy & 88.0 & 32.5 & 32.0 \\
\hline Mean & 97.52 & 29.33 & 32.84 \\
\hline L.S.D. $(0.05)$ & 3.02 & 3.73 & 8.94 \\
\hline
\end{tabular}


TABLE 8. Weight (g) of 100 seeds of pigeon pea groun at Fortuna, Puerto Rieo

\begin{tabular}{|c|c|c|c|c|}
\hline & & 100-seed weight & & \\
\hline & & -80 & $1980-81$ & $1981-82$ \\
\hline & First planting & Second planting & & \\
\hline 21 & 29.6 & 38.5 & 31.8 & 36.0 \\
\hline 24 & 32.1 & 35.6 & 28.1 & 33.7 \\
\hline 30 & 30.1 & 32.3 & 29.7 & 33.0 \\
\hline 43 & 29.3 & 32.5 & 29.0 & 32.6 \\
\hline 48 & 36.3 & 42.3 & 35.0 & 40.4 \\
\hline 49 & 30.1 & 37.5 & 32.4 & 35.9 \\
\hline 51 & 29.8 & 39.1 & 30.5 & 36.5 \\
\hline 53 & 30.5 & 34.5 & 32.0 & 35.5 \\
\hline 69 & 29.8 & 37.8 & 30.8 & 33.1 \\
\hline 76 & 26.5 & 27.8 & 26.0 & 30.1 \\
\hline $77-1$ & 29.3 & 34.4 & 31.9 & 33.0 \\
\hline 86 & 32.4 & 35.4 & 31.0 & 34.5 \\
\hline 92 & 29.1 & 30.9 & 30.3 & 33.1 \\
\hline 93 & 30.6 & 31.5 & 28.6 & 34.0 \\
\hline 97 & 32.3 & 29.5 & 30.8 & 37.8 \\
\hline 98 & 30.0 & 29.9 & 29.9 & 35.3 \\
\hline 100 & 29.3 & 32.4 & 30.3 & 32.7 \\
\hline $100-1$ & 38.0 & 34.3 & 28.7 & 34.9 \\
\hline 102 & 30.5 & 34.3 & 31.1 & 35.6 \\
\hline 109 & 30.8 & 38.5 & 30.7 & 35.9 \\
\hline 110 & 34.3 & 31.3 & 33.4 & 37.3 \\
\hline 118 & 29.1 & 31.0 & 28.7 & 31.3 \\
\hline 133 & 25.0 & 27.5 & 23.0 & 23.9 \\
\hline 135 & 32.8 & 34.5 & 29.1 & 33.5 \\
\hline 147 & 32,0 & 33.9 & 30.9 & 34.2 \\
\hline 148 & 29.6 & 35.6 & 31.2 & 30.2 \\
\hline 2B-Bushy & 31.3 & 31.5 & 29.3 & 31.2 \\
\hline Mean & 30.77 & 33.50 & 30.14 & 33.88 \\
\hline L.S.D. $(0.05)$ & 3.79 & 3.47 & 2.64 & 3.99 \\
\hline
\end{tabular}


J. Agric. Univ. P.R. voL. 72, No. 4, OCTOBER, 1988

TABLE 9.-Seeds per pod of pigeon pea grown at Fortuna, Puerto Rico

\begin{tabular}{|c|c|c|c|c|}
\hline \multirow[b]{2}{*}{ Genotype } & \multicolumn{2}{|c|}{$1979-80$} & \multirow[t]{2}{*}{$1980-81$} & \multirow[t]{2}{*}{$1981-82$} \\
\hline & First planting & Seeond planting & & \\
\hline 21 & 3.9 & 3.5 & 4.0 & 4.2 \\
\hline 24 & 3.5 & 3.6 & 3.5 & 3.3 \\
\hline 30 & 3.8 & 4.1 & 3.9 & 4.0 \\
\hline 43 & 4.1 & 3.7 & 3.7 & 4.2 \\
\hline 48 & 3.6 & 3.6 & 3.5 & 3.8 \\
\hline 49 & 3.6 & 3.3 & 3.7 & 4.1 \\
\hline 51 & 3.8 & 4.1 & 3.7 & 4.2 \\
\hline 53 & 3.7 & 3.6 & 3.8 & 4.0 \\
\hline 69 & 4.0 & 4.0 & 4.0 & 4.0 \\
\hline 76 & 4.2 & 4.1 & 4.4 & 4.3 \\
\hline $77-1$ & 3.7 & 3.8 & 4.1 & 4.2 \\
\hline 86 & 3.8 & 3.6 & 3.9 & 4.2 \\
\hline 92 & 3.9 & 4.2 & 4.0 & 4.2 \\
\hline 93 & 4.0 & 4.4 & 3.8 & 4.3 \\
\hline 97 & 4.2 & 4.5 & 4.4 & 4.2 \\
\hline 98 & 3.8 & 4.1 & 4.2 & 4.2 \\
\hline 100 & 4.1 & 4.6 & 4.2 & 4.4 \\
\hline $100-1$ & 4,2 & 3.9 & 3.9 & 4.0 \\
\hline 102 & 3.8 & 4.4 & 3.9 & 4.1 \\
\hline 109 & 3.8 & 4.1 & 4.1 & 4.3 \\
\hline 110 & 4.0 & 4.7 & 4.1 & 4.4 \\
\hline 118 & 4.1 & 3.8 & 4.2 & 4.0 \\
\hline 133 & 4,1 & 3.9 & 3.9 & 4.3 \\
\hline 135 & 3.9 & 3.8 & 3.6 & 4.3 \\
\hline 147 & 3.9 & 3.6 & 3.6 & 4.0 \\
\hline 148 & 4.0 & 3.7 & 3.8 & 4.3 \\
\hline 2B-Bushy & 3.8 & 3.7 & 3.6 & 4.1 \\
\hline Mean & 3.88 & 3.89 & 3.91 & 4.13 \\
\hline L.S.D.(0.05) & 0.35 & 0.35 & 0.27 & 0.45 \\
\hline
\end{tabular}


MORALES ET AL./PIGEON PEA

TABIE 10.-Seeds per pod of pigeon pea grown at Isabela, Puerto Rico

\begin{tabular}{cccc}
\hline Genotype & $1979-80$ & $1980-81$ & $1981-82$ \\
\hline 4 & 3.9 & 4.4 & 4.1 \\
29 & 4.1 & 4.5 & 4.4 \\
50 & 4.7 & 4.8 & 4.1 \\
60 & 4.0 & 4.8 & 4.3 \\
65 & 4.3 & 5.0 & 4.6 \\
79 & 4.4 & 4.9 & 4.3 \\
82 & 4.2 & 4.4 & 4.3 \\
83 & 4.0 & 4.5 & 4.0 \\
84 & 4.2 & 4.4 & 4.4 \\
98 & 4.2 & 4.8 & 4.2 \\
$100-1$ & 4.2 & 4.8 & 4.3 \\
109 & 4.1 & 4.7 & 4.3 \\
115 & 3.9 & 4.3 & 3.9 \\
120 & 4.0 & 4.7 & 4.3 \\
134 & 3.9 & 4.5 & 4.5 \\
135 & 4.0 & 4.4 & 4.2 \\
137 & 4.3 & 4.5 & 4.3 \\
147 & 4.1 & 4.4 & 4.2 \\
148 & 4.4 & 4.2 & 4.3 \\
151 & 4.4 & 4.6 & 4.4 \\
153 & 4.1 & 4.5 & 4.3 \\
$2 B-B u s h y$ & 3.6 & 4.1 & 3.9 \\
Mean & 4.10 & 4.55 & 4.26 \\
L.S.D. 0.05$)$ & 0.64 & 0.32 & NS \\
\hline
\end{tabular}

